\title{
Naturally-Formed Nanoscale Phase Separation in Epitaxially-Grown III-V Semiconductor Alloys
}

\author{
M. Ishimaru,* Y. Tanaka,* S. Hasegawa, ${ }^{*}$ H. Asahi, ${ }^{*}$ K. Sato, ${ }^{* *}$ and T. J. Konno** \\ * Institute of Scientific and Industrial Research, Osaka University, Ibaraki, Osaka 567-0047, Japan \\ ** Institute for Materials Research, Tohoku University, Sendai, Miyagi 980-8577, Japan
}

The introduction of structurally and/or compositionally modulated structures into semiconductor materials is of technological importance for adding desirable functionalities to electronic and optoelectronic devices. Spontaneous formation of nanostructures during crystal growth, the socalled self-assembly or self-organization, is one of the useful ways to introduce the modulated structure to materials in atomic scale. Here we demonstrate that nanoscale phase separation perpendicular to the growth direction can be realized in III-V semiconductor alloys.

TlGaInAsN/TIInP multiple quantum well structures were grown at $420{ }^{\circ} \mathrm{C}$ on $\operatorname{InP}(100)$ substrates by gas source molecular-beam epitaxy. Figure 1(a) shows a cross-sectional bright-field TEM image of the obtained specimen, viewed along the [110] direction. The TlGaInAsN (bright contrast) and TlInP (dark contrast) layers are alternatively stacked. The TIInP layers reveal uniform diffraction contrast, whereas a modulated structure exists in the TlGaInAsN layers. In the electron diffraction pattern (inset), satellite reflections are apparent on both sides of the Bragg reflections, indicating that the modulated structure is highly arranged. The modulation period was estimated to be $\sim 1 \mathrm{~nm}$. On the other hand, no modulation was observed in the image and diffraction pattern viewed along the [1-10] direction. That is, vertical quantum wells are formed in the TlGaInAsN layers. The modulated structure is also confirmed by HAADF-STEM observations [Fig. 1(b)]. From the HAADF-STEM and EDX analyses, it was found that the modulated structure consists of Ga- and Inrich regions, indicating that nanoscale phase separation occurs along the lateral direction [1].

We discuss the formation process of nanoscale phase separation based on a simple kinetic Ising model in which the rearrangement of atoms occurs at the topmost layer only and the atomic configuration is frozen-in when the surface is covered with a next monolayer [2]. In this model, pairwise atomic interactions up to the second nearest neighbors are considered [Figs. 2(a) and 2(b)]. From Monte Carlo simulations, it was found that the pairwise atomic interactions are defined as follows: $V_{1 \mathrm{~h}}<0, V_{1 \mathrm{~h}}{ }^{\prime}>0, V_{2 \mathrm{~h}}<0, V_{1 \mathrm{v}}>0$, and $V_{2 \mathrm{v}}<0$, where the positive and negative values prefer homonuclear (Ga-Ga and In-In) and heteronuclear (Ga-In) atomic pairs, respectively. When the growth temperature is low and the migration of atoms is suppressed, the ordering on the surface proceeds incompletely and homonuclear bonds are inevitably formed [Fig. 2(c)]. Once such homonuclear bonds are formed, the same atoms preferentially assemble on them in the next deposited monolayer due to the positive interaction $V_{1 \mathrm{v}}$ [Fig. 2(d)]. The epilayer thus obtained includes like-atom clusters which elongate along the growth direction [Fig. 2(e)].

References

[1] M. Ishimaru et al., Appl. Phys. Lett. 94, 153103 (2009).

[2] M. Ishimaru et al., Phys. Rev. B 51 (1995) 9707: 52 (1995) 5154: 54 (1996) 10814.

[3] A portion of this study was performed under the inter-university cooperative research program of the IMR, Tohoku University. 

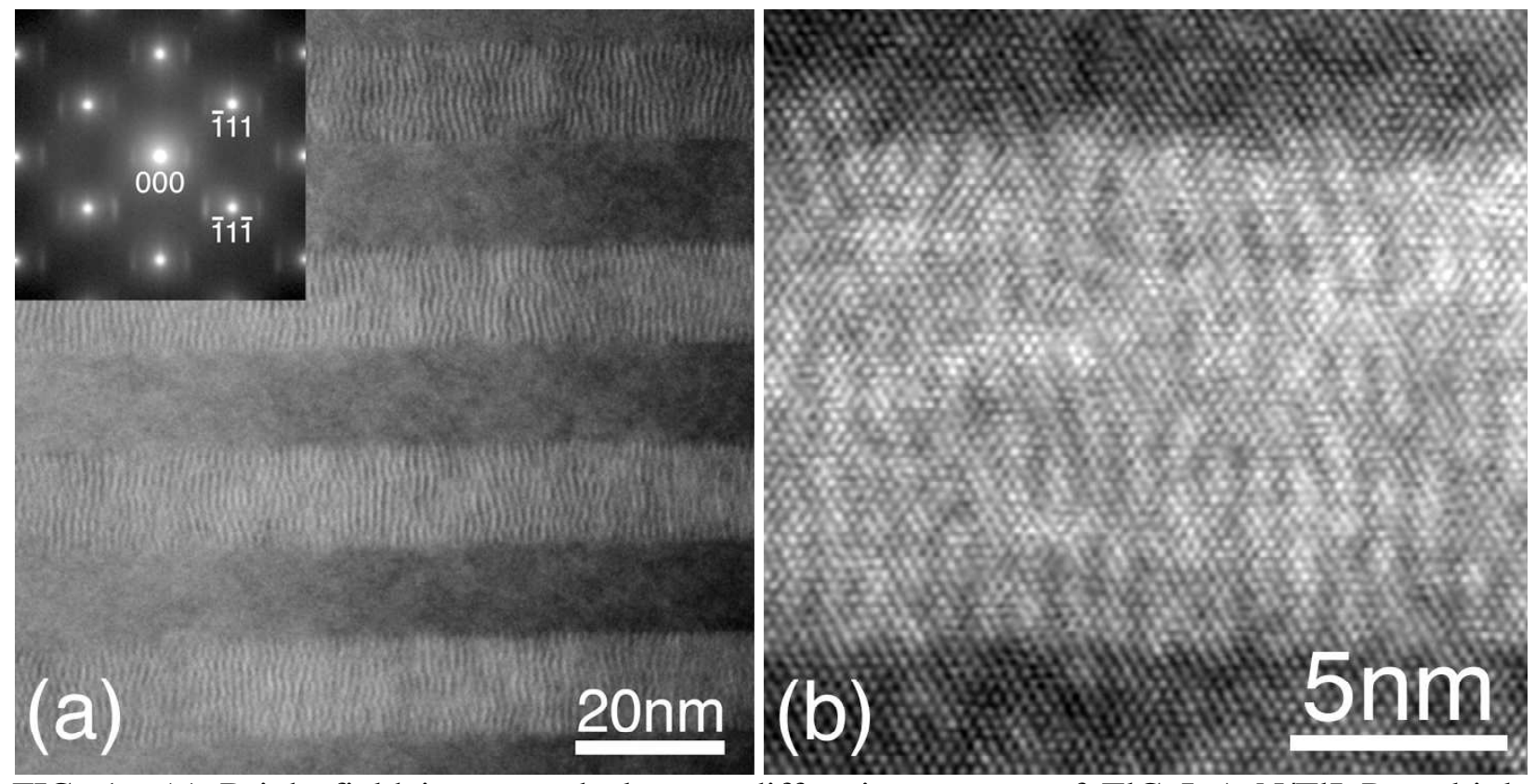

FIG. 1 (a) Bright-field image and electron diffraction pattern of TlGaInAsN/TIInP multiple quantum well structure. (b) HAADF-STEM image of TIGaInAsN layer.

(a)

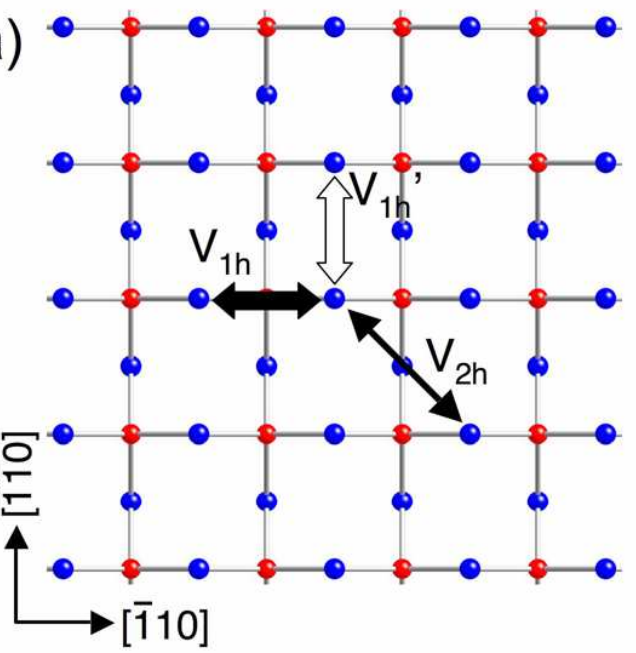

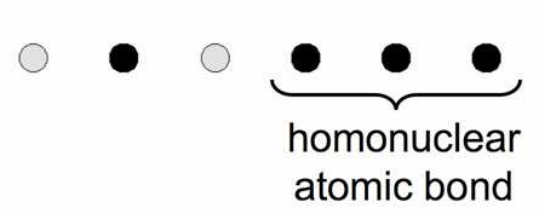

(c)

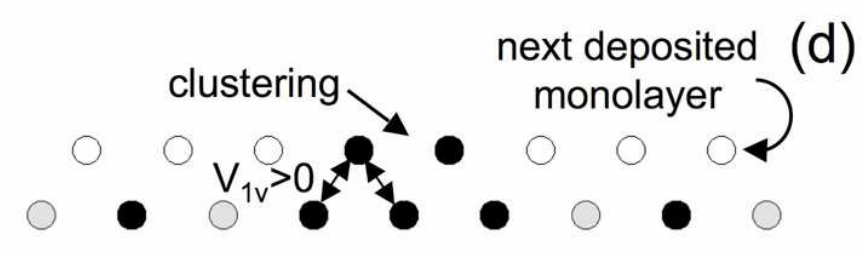

nanoscale phase separation perpendicular to the growth direction

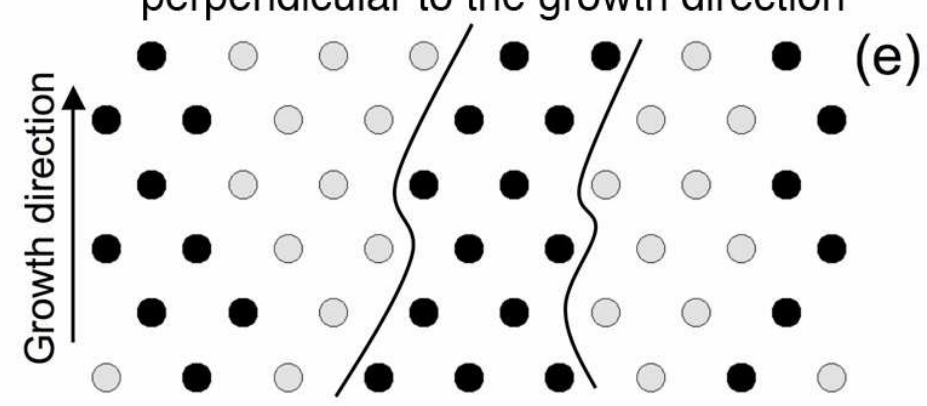

FIG. 2. (a,b) Pairwise atomic interactions at the topmost layer of an $\left(\mathrm{A}_{1-x} \mathrm{~B}_{x}\right)_{0.5} \mathrm{C}_{0.5}$ alloy. (a) Plan-view and (b) cross-sectional view. A and B (blue) are group-III elements and $\mathrm{C}$ (red) is a group-V element, or vise versa. (c-e) Formation process of like-atom chains along the growth direction. Only group-III elements are drawn, and black and gray circles correspond to A and B atoms, respectively 